\title{
GUI Design for Comparative Output Signal DFT and DTFT with LabVIEW
}

\author{
Dwi Astharini ${ }^{1}$, Tsaura Aulia ${ }^{2}$, Putri Wulandari ${ }^{3}$, Rahmat Alamtaha $^{4}$, and Rifqy Afisha ${ }^{5}$
}

\begin{abstract}
An application GUI (Graphical User Interface) is designed as a software simulation to compare the output signal of DFT and DTFT. DFT (Discrete Fourier Transform) and DTFT (Discrete Time Fourier Transform) are part of the digital signal processing. Digital signal processing is an analog signal processing method uses a mathematical technique to perform a transformation or retrieving information in digital form. One of the benefits of digital signal processing is to facilitate the representation of the signal, because the signal in digital form will be more visible, easily processed and has high accuracy. This GUI application designed using LabVIEW from National Instruments. LabVIEW is a software graphical programming or a block diagram. LabVIEW program known as VI or Virtual Instrument. The input signal in this application is a square signal.
\end{abstract}

Keywords-DFT, DTFT, Digital Signal Processing, LabVIEW, Virtual Instrument.

\section{INTRODUCTION}

$\mathbf{U}$ SER Interface is a tool that is used almost universally after it is widely known. The user interface that is known today is a design or implementation of a software designed to facilitate users. Users can control all objects such as giving several commands, replacing available variables, and managing what the user wants related to the object. The problem that often occurs is that the user interface is usually difficult to design, because of the demands for user convenience. The user interface is also known as GUI (Graphical User Interface). [1]

One of the tools of the GUI is LabVIEW. LabVIEW (Laboratory Virtual Instrumentation Engineering Workbench) is a graphical programming software from the National Instrument company. LabVIEW is designed to system engineering software for applications that require control, measurement and testing. In addition, LabVIEW can be used to design a system, control instrumentation and signal processing. [2]

In [3] uses LabVIEW to process signal analysis and processing frequency speed regulation of motor starting. Also, [5] uses LabVIEW for signal processing and analysis for Electroretinogram. LabVIEW tools in this paper is used to simulate ERG signals. In [9] the LabVIEW application is used to design the Fourier Transformation operation. It displays the spectrum of several signals such as sinusoidal, pulse train, Gaussian. There are also several windowing features such as Hanning, Hamming and Blackman-Harris.

This paper will discuss about a GUI design using LabVIEW from National Instrument. This GUI can display transformation signals using DFT and DTFT. The output of the DFT signal can be seen through the graph. For DFT signal output, there are two graphs these are magnitude graph and phase graph. For DTFT, the signal output can be seen also through the graph available on the GUI front panel. This paper makes it easy for users to be able to compare outputs from the DFT and DTFT signals. Considering that DFT can be calculated using FFT, this paper uses FFT tools for output signals in block diagrams.

\section{THEORY}

\section{A. Signal Processing}

In the world of signaling, the main problem for engineers is to analyze these signals and convert it into electrical signals such as microphones in communication techniques, blood pressure in the biomedical world, seismic transducers in science, etc. The old method used to analyze signals is to observe the signal in the time domain. Described by a French mathematician and physicist, Jean Baptiste Fourier, the signal can be represented as properly chosen sinusoidal waves. [9]

Fourier transformation is a tool used to analyze or process signals. Simply put, Fourier Transform is used to change the time domain into a frequency domain.[6] Changing the signal domain will make the analysis easier. In terms of signal processing, Fourier Transforms can be used to analyze signals and systems. When it used signal analysis the signal spectrum will be generated. When it used for system analysis, a system frequency response will be generated. The signal can be continuous or discrete, it can also be periodic or aperiodic. In Fourier transform the combination of these two features produces four categories. Below will explain the four categories:

1) Aperiodic-Continuous: these signal has a range of positive and negative infinity without repeating in a periodic pattern. This type of signal is called the Fourier Transform.

2) Periodic-Continuous: all signals waveform that repeat the pattern of negative and positive infinity, commonly called Fourier series.

3) Aperiodic-Discrete: are discrete signals that not repeats according to the pattern (aperiodic). This type is usually called Discrete Time Fourier Transform (DTFT).

4) Periodic-Discrete: are discrete signals that repeats according to the pattern (periodically) sometimes called Discrete Fourier Series but is most often called the Discrete Fourier Transform (DFT). 


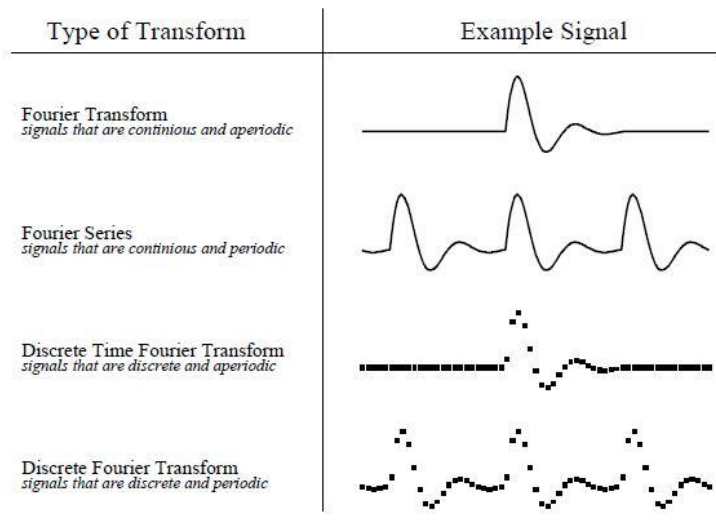

Fig. 1. Illustration of four Fourier Transforms. The signal can be continuous or discrete, it can also be periodic or aperiodic.

\section{a. Discrete Fourier Transform (DFT)}

The input signal is usually in the time domain, because many types of signals that entering the DFT consists of samples taken at regular time intervals. All types of sample data can be entered into DFT, depending on how it was acquired. When we have a time domain, the process becomes a frequency domain called decomposition, analysis, forward DFT or simply, DFT. If we have frequency domain data, the process into a time domain is called synthesis or inverse DFT. Assume that we have a signal $\{x[n]\}_{n=0}^{N-1}$ The DFT of a the signal is a sequence $X[k]$ for $k=0, \ldots, N-1$, can be given as

$$
X[k]=\sum_{n=0}^{N-1} \mathrm{x}[\mathrm{n}] e^{-j 2 \pi n k / N}
$$

The Inverse Discrete Fourier Transform (IDFT):

$$
x[n]=\frac{1}{N} \sum_{n=0}^{N-1} X[k] e^{j 2 \pi n k / N}, n=0,2, \ldots N-1
$$

The most efficient algorithm for calculating DFT is Fast Fourier Transform (FFT). The time taken to evaluate a DFT on a computer depends principally on the number of multiplications involved. DFT needs $\mathrm{N}^{2}$ multiplications. FFT only needs $\mathrm{Nlog}_{2}(\mathrm{~N})$. The term fast by using the FFT formulation is much faster than the previous Fourier Transfrom calculation method. The FFT technique requires about 10,000 mathematical operations for data with values, which are 100 times faster than previous calculation techniques. With this FFT discovery and personal computer development, FFT techniques in data analysis became popular, and is one of the standard methods in data analysis.

\section{b. Discrete Time Fourier Transform (DTFT)}

Discrete Time Fourier Transform is a continuous and periodic function of frequency, whereas DFT is a discrete function. DFT provides a discrete sample of continuous DTFT signals. Let us now sample the DTFT $X(f)$ at equispaced intervals which are apart by $1 / \mathrm{N}$ to obtain

$$
X^{\prime}\left(\frac{k}{N}\right)=\sum_{n=0}^{N-1} x[n] \exp \left(-j 2 \pi \frac{k}{N} n\right)
$$

Let us denote:

Thus,

$$
X[k]=X^{\prime}\left(\frac{k}{N}\right)
$$

$$
X[k]=\sum_{n=0}^{N-1} x[n] \exp \left(-j 2 \pi \frac{k}{N} n\right), n=0 \leq k \leq N-1
$$

\section{DESAIN APPLICATION}

GUI-based application will display the output signal DFT and DTFT transformation. In this application, there are three parts sub block, namely the introduction, process, and help. Front view of our application is shown in figure 3.

Sub-block both there is a process of simulation. On this block shown a graph of signal information and the signal DFT and DTFT transformation. Here is a picture of the front view of the simulation process menu is shown in Figure 3.

In menu process there is also a control variable to regulate the information signal. Variables set are the frequency, amplitude, and phase of the signal. Here is the view out of the control variables for signal information.

Using VI technology, we design the flow chart of graphical applications. Briefly, the figure 5 shows the process rather than an application made by us. The first process is to run the program marked with start. Then, the next path is found a switch to enter the case. If the case is correct, the program will continue and if the case is wrong, the program will stop. For true cases, the program continues by entering the amount of information signal. Inputs are amplitude, phase and frequency. After the information signal variable has been inputted, the process enters the square signal generator. Then there are three channels, namely information signal display, DFT signal processing and DTFT signal processing. For the DTFT signal process, it immediately comes out in the form of a graph. There are two graphs for processing the DFT signal, namely magnitude and phase graphs.

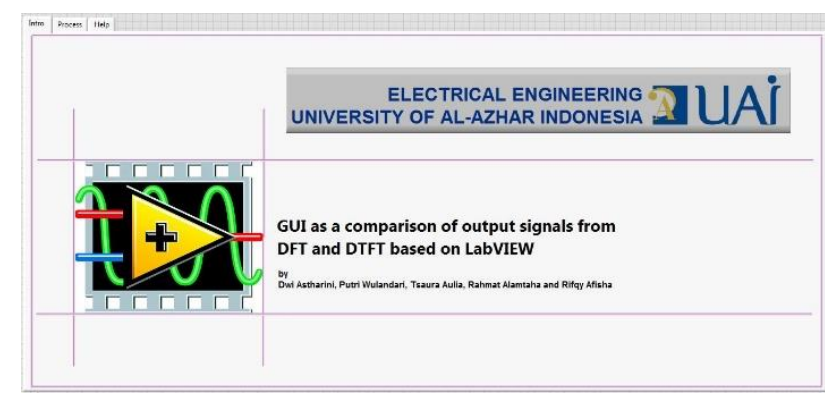

Fig. 2. The front display simulation applications

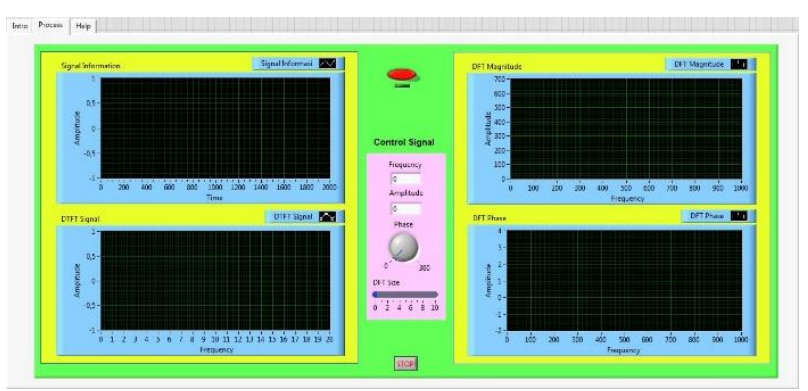

Fig. 3. Views on the Menu Process for Simulation. 


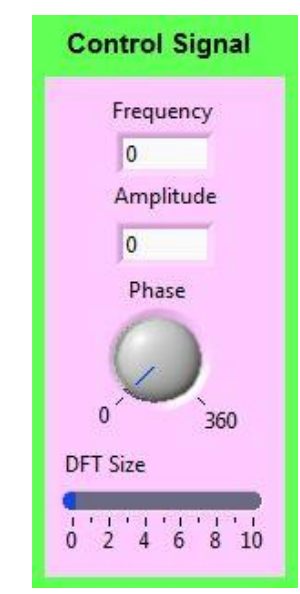

Fig. 4. Variable Control Signal Information

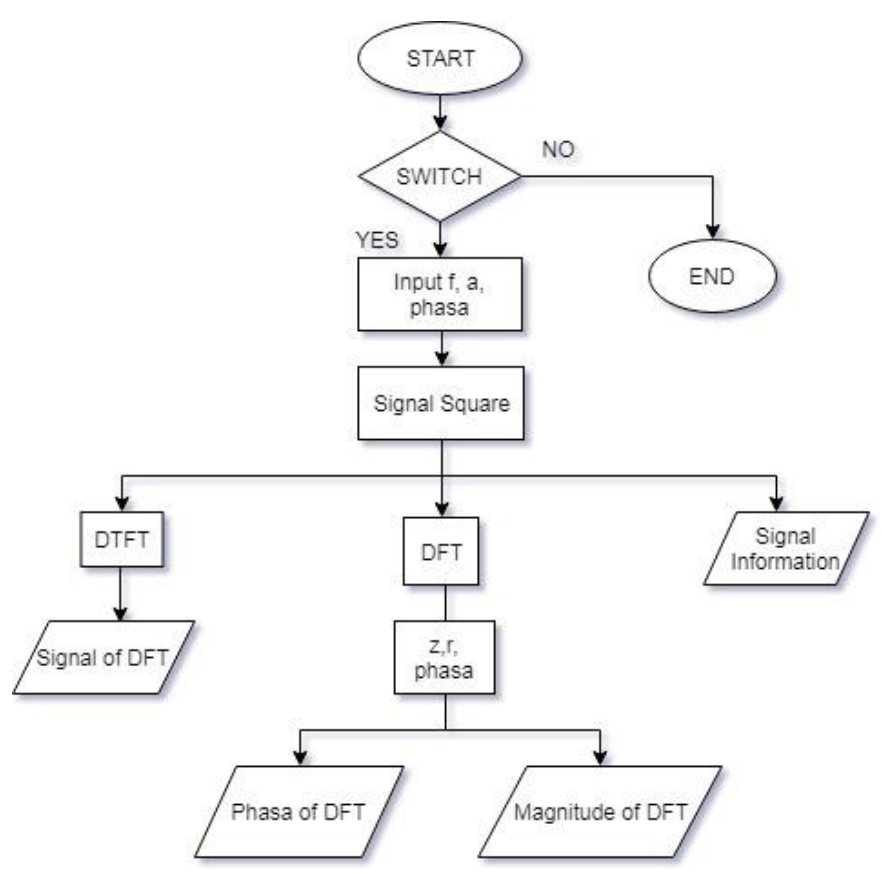

Fig. 5. Flow of Application

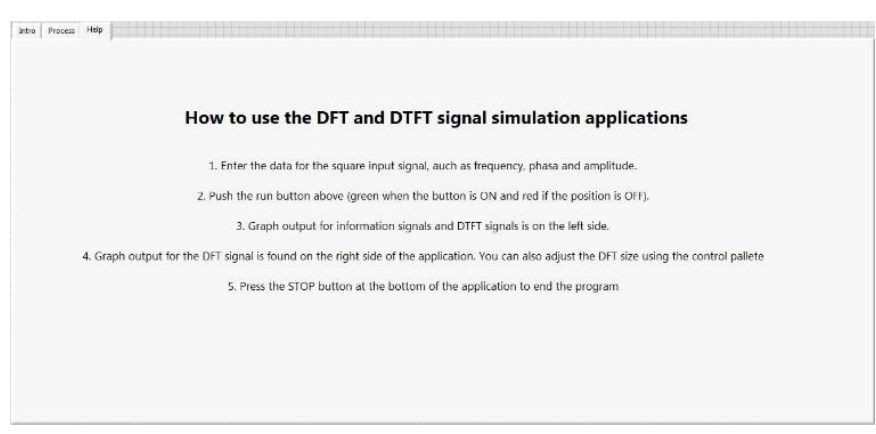

Fig. 6. Help menu on the last tab of application

For the third menu there is a menu Help that tells how to use this simulation application. The view from the help menu of application is shown in Figure 6

\section{RESUlT}

Using this signal simulation application, we did an experiment with setting the amount of information signal with amplitude of 2, the frequency of $100 \mathrm{~Hz}$ and an angle of 50 , and the DFT size is 30 . Here is a view of the control variables that have been set:

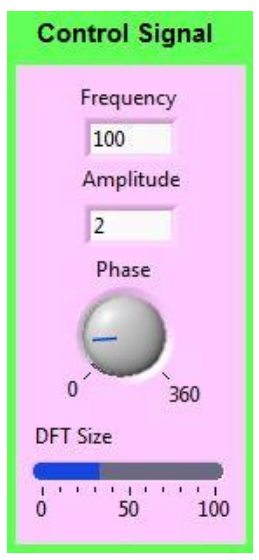

Fig. 7. Controlled Signal Information

The results obtained are information signals (square wave), magnitude and phase signals from DFT transformations, and DTFT transformation signals (using DFT approach). The view of the results of running programs is shown in figure 8 11.

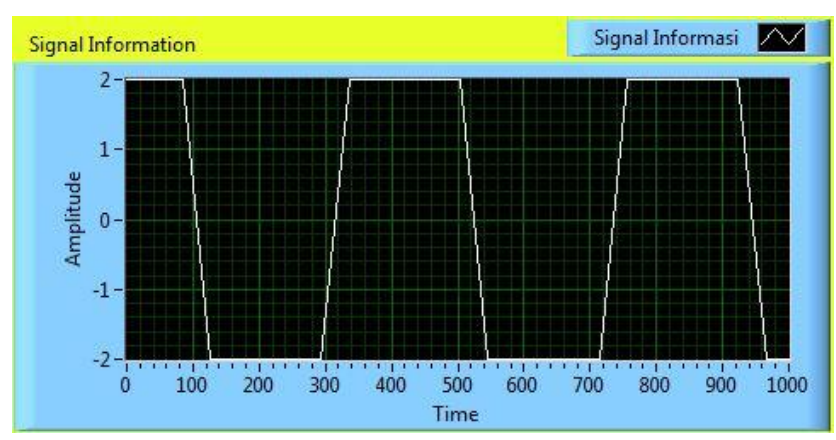

Fig. 8. Display of Signal Information

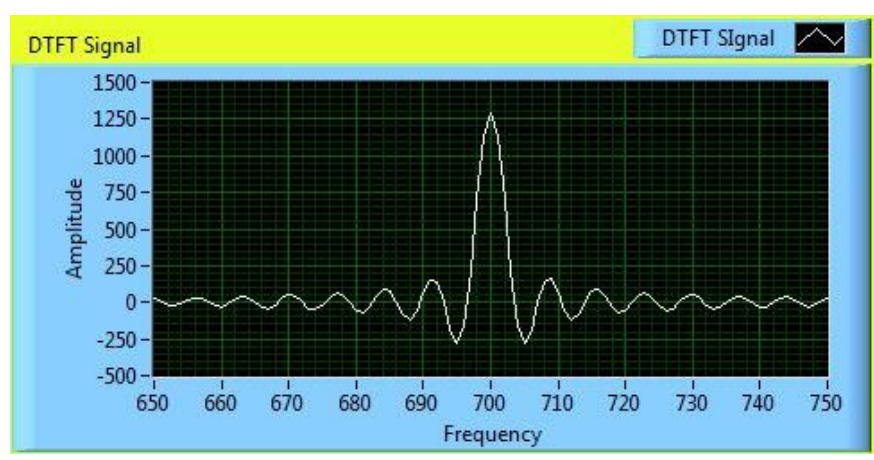

Fig. 9. Display of Signal DTFT (using DFT approach) 


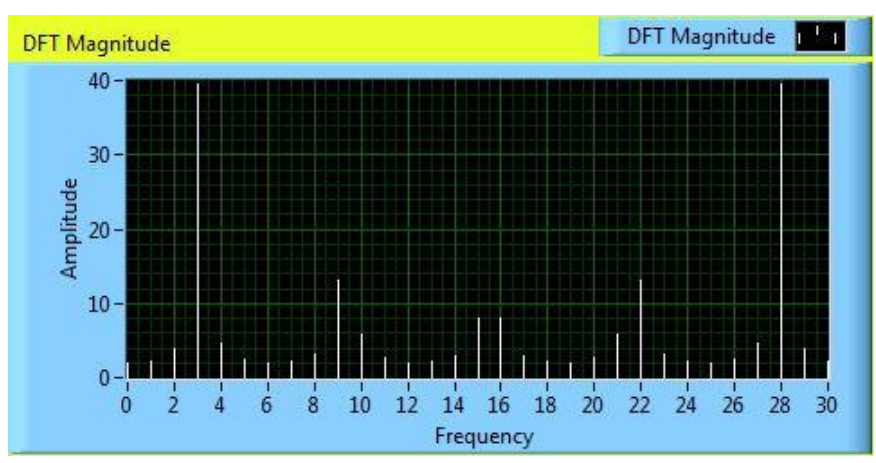

Fig. 10. Display Magnitude of DFT

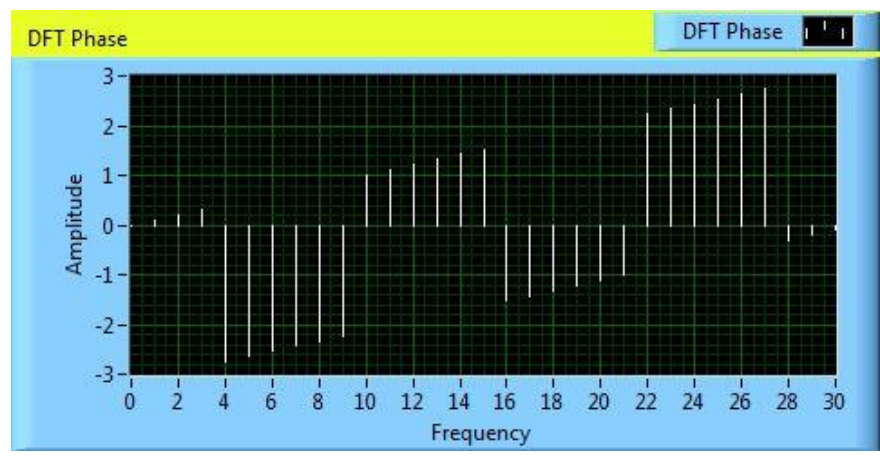

Fig. 11. Display Phasa of DFT

\section{RESUlT}

Using LabVIEW as a virtual instrument can facilitate the signal processing become faster. With this graphical software, can be made depending on the desired user needs with an output format is .exe. The results obtained are graph of information signals, magnitude and phase signals from DFT transformations, and DTFT transformation signals (via DFT approach). The tools in LabVIEW that used are FFT (Fast Fourier Transform) considering that DFT can be calculated using FFT and the results from DTFT can be done by DFT approach. With this application, users can set information signal variables in the form of amplitude, phase and frequency. Besides, it can also be set the large of DFT.

\section{REFERENCES}

[1] B. A. Myers, Graphical User Interface Programming CRC HANDBOOK OF COMPUTER SCIENCE AND ENGINEERING 2nd, LLC: CRC Press, 2004.

[2] D. E. Seborg, T. F. Edgar and D. A. Meilichamp, Process Dynamics and Control, 2nd Edition, New York: Wiley, 2004.

[3] H. Guo and J. Wu, "Application of Virtual Instrument LabVIEW in Variable," TELKOMNIKA Indonesian Journal of Electrical Engineering, vol. XII, no. 8, pp. 6119-6126, 2014.

[4] U. and M. S. Panse, "Electroretinogram (ERG) Signal Processing \& Analysis in LabVIEW," Advances in Medical Informatics, vol. I, no. 1, pp. 1-5, 2011.

[5] S. Dang, R. Kakimzhanov, M. Zhang and A. Gholamzadeh, "Smart Grid-oriented Graphical User Interface Design and Data Processing Algorithm Proposal Based on LabVIEW," International Conference on Environment and Electrical Engineering, vol. XIV, no. 1, pp. 47994660, 2014.

[6] P. Avitabile, J. Hodgkins, T. V. Zandt, A. Butland and D. Nickgorski, "INNOVATIVE TEACHING OF FOURIER SERIES USING LABVIEW," in American Society for Engineering Education Annual Conference \& Exposition, Massachusetts USA, 2006.

[7] T. Tasner, D. Lovrec, Tasner, Francisek and J. Edler, "COMPARISON OF LabVIEW AND MATLAB FOR SCIENTIFIC RESEARCH," Annals of Faculty Engineering Hunedoara - International Journal of Engineering, vol. X, no. 3, pp. 1584-1673, 2012.

[8] R. R. Serrezuela, A. F. Chavarro, M. A. T. Cardozo, A. G. R. Caicedo and C. A. Cabrera, "AUDIO SIGNALS PROCESSING WITH DIGITAL FILTERS IMPLEMENTATION USING MYDSP," ARPN Journal of Engineering and Applied Sciences, vol. XII, no. 16, pp. 4848-4853, 2017.

[9] L. SEVGI and C. ULUISIK, "A Labview-Based Virtual Instrument for Engineering Education: A Numerical Fourier Transform Tool," Turk $J$ Elec Engin, vol. XIV, no. 1, pp. 129-152, 2006.

[10] M. A. A. Kasim, C. Y. Low, M. A. Ayub, N. A. C. Zakaria, M. H. M. Saleh, K. Johar and H. Hamli, "User-Friendly LabVIEW GUI for Prosthetic Hand Control using Emotiv EEG Headset," IEEE International Symposium on Robotics and Intelligent Sensors, vol. 105, pp. 276-281, 2016. 